\title{
Emergency biventricular assist device implantation for acute cardiopulmonary failure in a patient with COVID-19
}

\author{
Nnamdi Nwaejike ${ }^{1}$, Timothy Strang ${ }^{1}$, Miguel Garcia ${ }^{1}$, Michael Charlesworth ${ }^{1}$, Steven \\ Shaw $^{1}$, and James Barnard ${ }^{1}$ \\ ${ }^{1}$ Wythenshawe Hospital
}

August 10, 2020

\begin{abstract}
Severe COVID-19 is a multisystem inflammatory disorder and knowledge and experience with severe acute respiratory failure in infected patients has grown considerably since reports of the first few cases. Little is known about the effect of SARS-CoV-2 on the heart, and there has been a suggestion from published literature that fulminant cardiac failure with or without respiratory failure may occur several weeks following infection. A young man presented after a recent viral illness. He was found to be in severe cardiogenic shock and was implanted with an emergency biventricular assist device, which also incorporated an extracorporeal membrane oxygenator. He stabilised soon thereafter and despite an intracerebral haemorrhage, which resolved, and bleeding into the trachea following percutaneous tracheostomy, he survived to explant and was successfully stepped down to a rehabilitation unit on postoperative day 50. He tested positive for SARS-CoV-2 antibodies when the test became available on postoperative day 33. We envisage there will be many more such presentations of acute COVID-19-associated cardiogenic shock and we recommend clinicians consider this diagnosis when presented with an acutely unwell patient with an unclear diagnosis, following a viral illness. These patients should be discussed as early as possible with a transplant/mechanical circulatory support team.
\end{abstract}

\section{Introduction}

Current knowledge on the use of extracorporeal membrane oxygenation for patients with severe refractory respiratory, cardiac and cardiopulmonary failure associated with COVID-19 is limited to case reports and small observational case series [1]. The clinical presentation of myocarditis associated with COVID-19 is variable, and fulminant myocarditis is usually associated with ventricular arrhythmias and heart failure within 2-3 weeks of contracting SARS-CoV-2 [2]. Early signs may resemble those of septic shock, with tachycardia, pyrexia, hypotension and mottled peripheries [3]. Little is known about how severe cases should be managed, and the role mechanical circulatory support should play is unclear $[4,5]$.

On the one hand, patients with fulminant myocarditis and cardiopulmonary failure will likely not survive without a high-level intervention such as venoarterial extracorporeal membrane oxygenation (VA-ECMO) or biventricular assist device (BiVAD) implantation. Nevertheless, such interventions remain a controversial area of practice due to unresolved questions about: patient selection; the balance of benefit and risk; and the distributive justice of resources [6]. We present the first case of a COVID-19 patient who presented to our institution with profound cardiopulmonary failure who was resuscitated and managed successfully to explant and recovery with a BIVAD as well as an extracorporeal membrane oxygenator.

\section{Report}

A 39-year-old, previously healthy male of Black African ethnic origin presented to the Emergency Department on the $25^{\text {th }}$ of April 2020 with fever and abdominal pain after a recent viral illness. Chest radiographs showed typical ground-glass changes indicative of viral pneumonia (Fig. 1A). A computer tomography (CT) 
scan showed acute pancreatitis, small bilateral pleural effusions, lung consolidation, associated reactive lymphadenopathy and features indeterminate for COVID-19 (Fig. 1B). Echocardiography revealed significant impairment of biventricular function. Nasal and pharyngeal swabs, bronchoalveolar lavage fluid, and sputum were tested for common respiratory viruses, and the results were all negative. Two SARS-CoV-2 antigen swab tests were also negative. He was referred to our team for emergency assessment with a view to mechanical circulatory support.

The patient arrived intubated and ventilated, with a temperature of $38.3^{\circ} \mathrm{C}$. He required an $\mathrm{FiO}_{2}$ of 1.0 and his peak airway pressure was $>35 \mathrm{cmH}_{2} \mathrm{O}$. He was receiving high-dose vasopressors and inotropes and the clinical picture was one of severe acute cardiopulmonary failure. He had a blood picture in keeping with hyperinflammation, with a serum ferritin of $2316 \mu \mathrm{g} . \mathrm{l}^{-1}$ and a CRP of $424 \mathrm{mg} . \mathrm{l}^{-1}$. His Troponin $\mathrm{T}$ was $616 \mathrm{ng} . \mathrm{l}^{-1}$ and his fibrinogen was $8.83 \mathrm{~g}^{-\mathrm{l}^{-1}}$. He was taken straight to the operating theatre for a transoesophageal echocardiographic examination with a view to BiVAD implantation. This confirmed severe biventricular failure(Video 1) with an ejection fraction of 0.31 , and with no structural intra-cardiac lesions or abnormalities.

Extracorporeal biventricular assist device therapy with two CentriMag pumps was established following

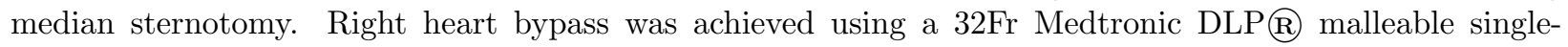

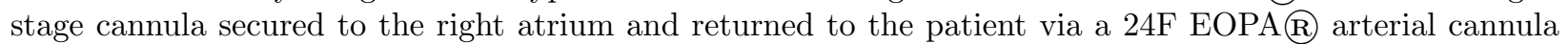
(pulmonary artery cannula). Left heart bypass was achieved by inserting a CentriMag ${ }^{\mathrm{TM}}$ 34Fr Drainage cannula in the left ventricular apex and returned to the patient via a $24 \mathrm{~F}$ EOPA@) arterial cannula (aortic cannula) (Video 2). We were unable to take myocardial biopsies intra-operatively due to the urgency of the procedure. An extracorporeal membrane oxygenator (sweep 1:1) was included in the left sided circuit due to deteriorating lung function. He was subsequently placed on lung rest settings (inspiratory pressure $20 \mathrm{cmH}^{2} \mathrm{O}$, PEEP $10 \mathrm{cmH}_{2} \mathrm{O}$, respiratory rate of $10 \mathrm{~min}^{-1}$ ). The flow rates on each circuit were set to 2.2 l. $\mathrm{min}^{-1} \cdot \mathrm{m}^{-2}$ and the acidosis and serum lactate levels resolved over the next 12 hours.

The patient was reexplored with the evacuation of a pericardial haematoma on postoperative day 4 . On postoperative day 11, a CT brain scan detected an intraparenchymal haemorrhage in the left parietal lobe deep white matter with cyst surrounding vasogenic oedema. There was no midline shift. A repeat CT brain on postoperative day 37 showed complete resolution of the haemorrhagic component and residual focal low attenuation.

By postoperative day 17, he had stabilised on the ventilator with good tidal volumes, and the oxygenator was removed from the left side of the circuit. He underwent a percutaneous tracheostomy on postoperative day 22 as per national guidelines [7]. On postoperative day 33, SARS-CoV-2 antibody tests became widely available in the UK, and he tested positive. He continued to make good progress; lung compliance returned to normal with improving ventilation and oxygenation (Fig. 1C), and his BiVAD circuits were explanted on postoperative day 44, with the heart supported on milrinone for 48 hours (Video 3) .

On postoperative day 50, the patient was sitting out of bed but still ventilator dependent on continuous positive pressure ventilation via a tracheostomy. He had global peripheral muscle weakness but was progressing well with physical therapy. He transferred out to a step-down unit in another hospital for continued rehabilitation (Fig. 1D).

\section{Discussion}

We report the successful treatment of a patient with probable COVID-19 induced cardiopulmonary failure, due to fulminant myocarditis, using a BIVAD with an extracorporeal membrane oxygenator. To our knowledge, there is only other one such case reported in the literature, and this patient died of a secondary bacterial infection on postoperative day 33 and prior to explantation [3]. Although VA-ECMO could also be considered and has been reported in the literature [8], this mode of support is not an effective long-term strategy and may lead to stasis of blood flow in the pulmonary circulation.

The clinical presentation was highly suspicious for COVID-19 despite negative SARS-CoV-2 antigen tests at 
the height of the UK component of the COVID-19 worldwide pandemic. Due to the initial unavailability of testing, he was only confirmed to be SARS-CoV-2 antibody positive four weeks post-BIVAD implantation. We conclude that he likely contracted COVID-19 before presentation to hospital, then subsequently succumbed to an overwhelming systemic inflammatory response, which resulted in cardiopulmonary failure.

Coagulopathy is a recognised complication of mechanical circulatory support and COVID-19 [9]. Initially, the patient was coagulopathic, so systemic heparinisation was delayed until postoperative day 3 . An intracerebral bleed was detected on postoperative day 11 which later resolved. A significant bleed within the trachea complicated the percutaneous tracheostomy, which required packing, re-intubation and a return to theatre for exploration and resolution. Heparin infusions were discontinued for prolonged periods due to the intracerebral bleed, tracheostomy bleed and thrombocytopenia. Heparin-induced thrombocytopenia screen tests were negative and the thrombocytopenia resolved with discontinuation of the BIVAD and with continued platelet transfusions.

Our report documents one of the first reported successful cases of a patient with COVID-19 induced cardiopulmonary failure with BIVAD mechanical circulatory support. The outcome here suggests that COVID-19 patients with acute cardiogenic shock may be successfully managed with a BIVAD, but there are several challenges that may be encountered as well as significant resources required. Patient selection can be challenging and Chow et al. recommend that identification of potential clinical scenarios leading to cardiogenic shock and circumstances unique to COVID-19 may facilitate decision-making, ideally by a multidisciplinary team that includes representation of cardiac surgery, cardiology, intensive care, anaesthesia, as well as advanced heart failure and transplant physicians [10].

In this patient, we applied a proven emergency therapy to treat a patient with near fatal biventricular failure. The aetiology of the condition was not known at the initiation of BiVAD due to challenges in the availability of the SARS-CoV-2 antibody test. It is likely that cases of COVID-19-associated cardiogenic shock present several weeks after the initial infection, test negative for the SARS-CoV-2 antigen on presentation, and that such cases will continue to be seen in the future. We recommend that all healthcare professionals keep this diagnosis in mind when presented with a young, acutely unwell patient with cardiogenic shock of unknown aetiology, and refer such patients to their local transplant/mechanical circulatory support service.

\section{Acknowledgements}

We thank our critical care nursing staff, technicians, anaesthetists and especially our perfusionists, T. Quinton and B. Pate. No conflicts of interest and no external funding.

\section{References}

1. Zochios V, Brodie D, Charlesworth M, Parhar KK. Delivering extracorporeal membrane oxygenation for patients with COVID-19: what, who, when and how? Anaesthesia 2020; 75 : 997-1001.

2. Siripanthong B, Nazarian S, Muser D et al. Recognizing COVID-19-related myocarditis: The possible pathophysiology and proposed guideline for diagnosis and management. Heart Rhythm 2020 Epub 5 May. doi.org/10.1016/j.hrthm.2020.05.001.

3. Zeng JH, Liu YX, Yuan J et al. First case of COVID-19 complicated with fulminant myocarditis: a case report and insights. Infection2020 Epub 10 Apr. doi.org/10.1007/s15010-020-01424-5.

4. Pham DT, Toeg H, De Paulis R, Atluri P. Establishment and management of mechanical circulatory support during the COVID-19 pandemic. Circulation 2020; 142 : 10-3.

5. MacLaren G, Fisher D, Brodie D. Preparing for the most critically ill patients with COVID-19: the potential role of extracorporeal membrane oxygenation. Journal of the American Medical Association 2020;323 : $1245-6$. 
6. Charlesworth M, Garcia M, Head L et al. Venoarterial extracorporeal membrane oxygenation for postcardiotomy cardiogenic shock-A six-year service evaluation. Artificial Organs 2020; 44 : 709-16.

7. McGrath BA, Ashby N, Birchall M et al. Multidisciplinary guidance for safe tracheostomy care during the COVID-19 pandemic: the NHS National Patient Safety Improvement Programme (NatPatSIP). Anaesthesia2020 Epub 12 May. doi.org/10.1111/anae.15120

8. Salamanca J, Díez-Villanueva P, Martínez P et al. COVID-19 "Fulminant myocarditis" successfully treated with temporary mechanical circulatory support. Journal of the American College of Cardiology: Cardiovascular Imaging 2020 Epub 11 May. doi.org/10.1016/j.jcmg.2020.05.003.

9. Thachil J, Agarwal S. Understanding the COVID-19 coagulopathy spectrum. Anaesthesia 2020 Epub 21 May. doi.org/10.1111/anae.15141.

10. Chow J, Alhussaini A, Calvillo-Argüelles O, Billia F, Luk A. Cardiovascular collapse in covid-19 infection: the role of venoarterial extracorporeal membrane oxygenation (VA-ECMO). CJC Open 2020;2 : 273-7.

Figure legends

Figure 1A Chest radiograph on presentation showing lung consolidation

Figure 1B CT on presentation showing lung consolidation

Figure 1C Chest radiograph showing the improvement seen with the BiVAD

Figure 1D Chest radiograph on the day of discharge to the step-down unit

Video 1 - Pre-BIVAD short-axis Transoesophageal echocardiography

Video 2 - Transoesophageal echocardiogram showing Left ventricular Apical Cannula outflow

Video 3 - Post-BIVAD short-axis Transoesophageal echocardiography

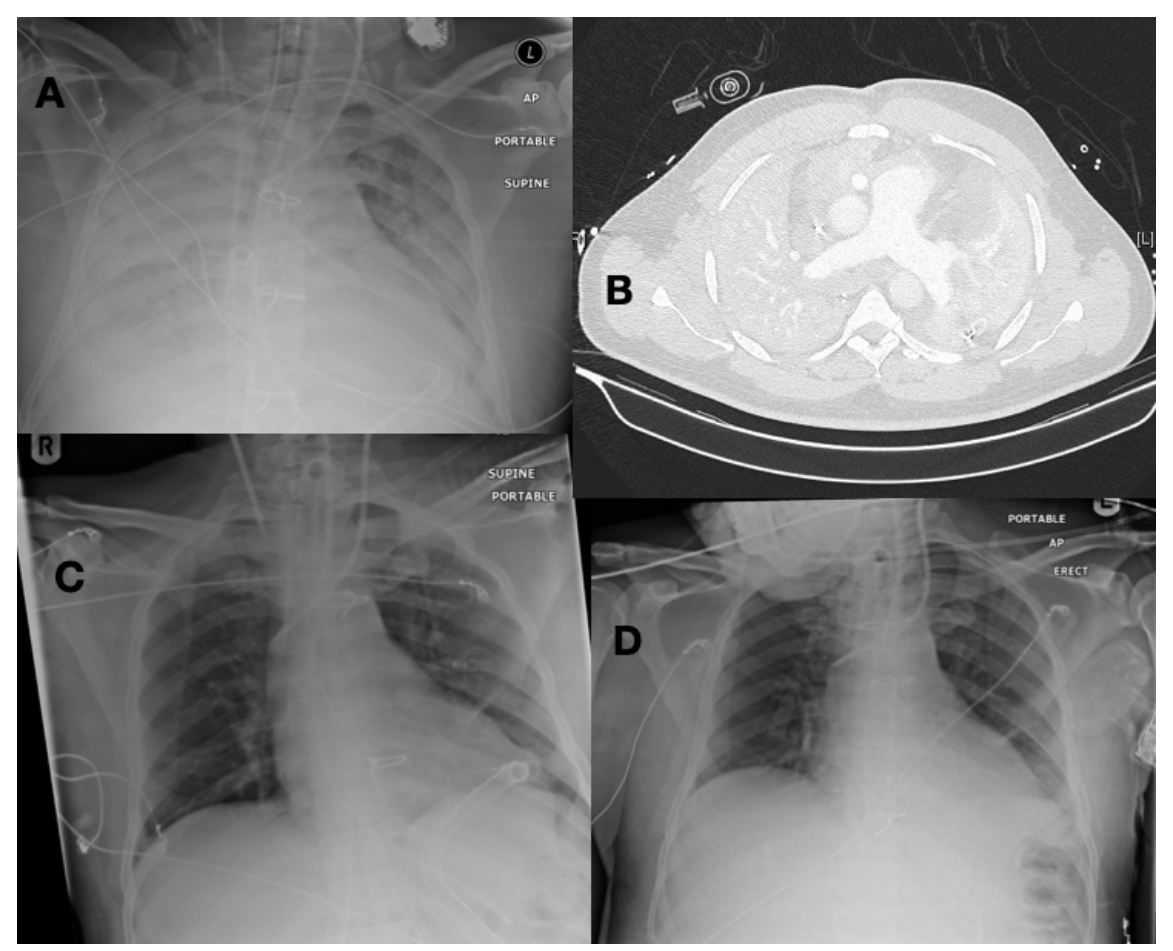

